\title{
Supporting Information for "Large-scale Surface Shape Sensing with Learning-based Computational Mechanics"
}

\author{
Kui Wang ${ }^{1}$, Chi Hin Mak ${ }^{1}$, Justin Di-Lang Ho${ }^{1}$, Zhi-Yu Liu ${ }^{1}$, Kam Yim Sze ${ }^{1}$, Kenneth \\ K.Y. Wong ${ }^{1}$, Kaspar Althoefer ${ }^{1}$, Yunhui Liu ${ }^{1}$, Toshiko Fukuda ${ }^{1}$, and Ka-Wai Kwok ${ }^{1}$ \\ ${ }^{1}$ Affiliation not available
}

July 6,2021

\begin{abstract}
Proprioception, the ability to perceive one's own configuration and movement in space, enables organisms to safely and accurately interact with their environment and each other. The underlying sensory nerves that make this possible are highly dense and use sophisticated communication pathways to propagate signals from nerves in muscle, skin and joints to the central nervous system wherein the organism can process and react to stimuli. In a step forward to realize robots with such perceptive capability, we propose a flexible sensor framework that incorporates a novel hybrid modeling strategy, taking advantage of computational mechanics and machine learning. We implement the sensor framework on a large, thin and flexible sensor that transforms sparsely distributed strains into continuous surface shape. Finite element (FE) analysis is utilized to determine sensor design parameters, while an FE model is built to enrich the morphological data used in the supervised training to achieve continuous surface reconstruction. A mapping between the local strain data and the enriched surface data is subsequently trained using ensemble learning. This hybrid approach enables real-time, robust and high-order surface shape reconstruction. The sensing performance is evaluated in terms of accuracy, repeatability, and feasibility with numerous scenarios, which has not been demonstrated and reported on such a large-scale (A4-paper-size) sensor before.
\end{abstract}

\section{Results and Discussion}

An A4-sized $(210 \times 297 \mathrm{~mm})$ flexible surface shape sensor (Figure. 1) was developed through our FEintegrated framework (Figure. S1). FE modeling is utilized for testing design parameters of the sensor prior to fabrication, wherein parameters such as sensor thickness, sensing element placement can be tuned depending on the intended application. The surface shape sensor utilized 29 sparsely distributed FBGs as the underlying sensing elements for measuring strain, which were subsequently used as inputs to train a model to reconstruct the 3D surface morphology of the shape sensor. A demonstration of the shape sensor's response to different external stimuli is presented in Videos $\mathbf{1 ,} \mathbf{2}$, including shape reconstruction during hands-on deformation, and impact from water jet and small projectile beads. Shape reconstruction accuracy, repeatability and hysteresis tests were also performed on the developed shape sensor. The resulting performance is achieved through our hybrid approach of FE-based data enrichment and ensemble learning method, with details described in the sections below. 


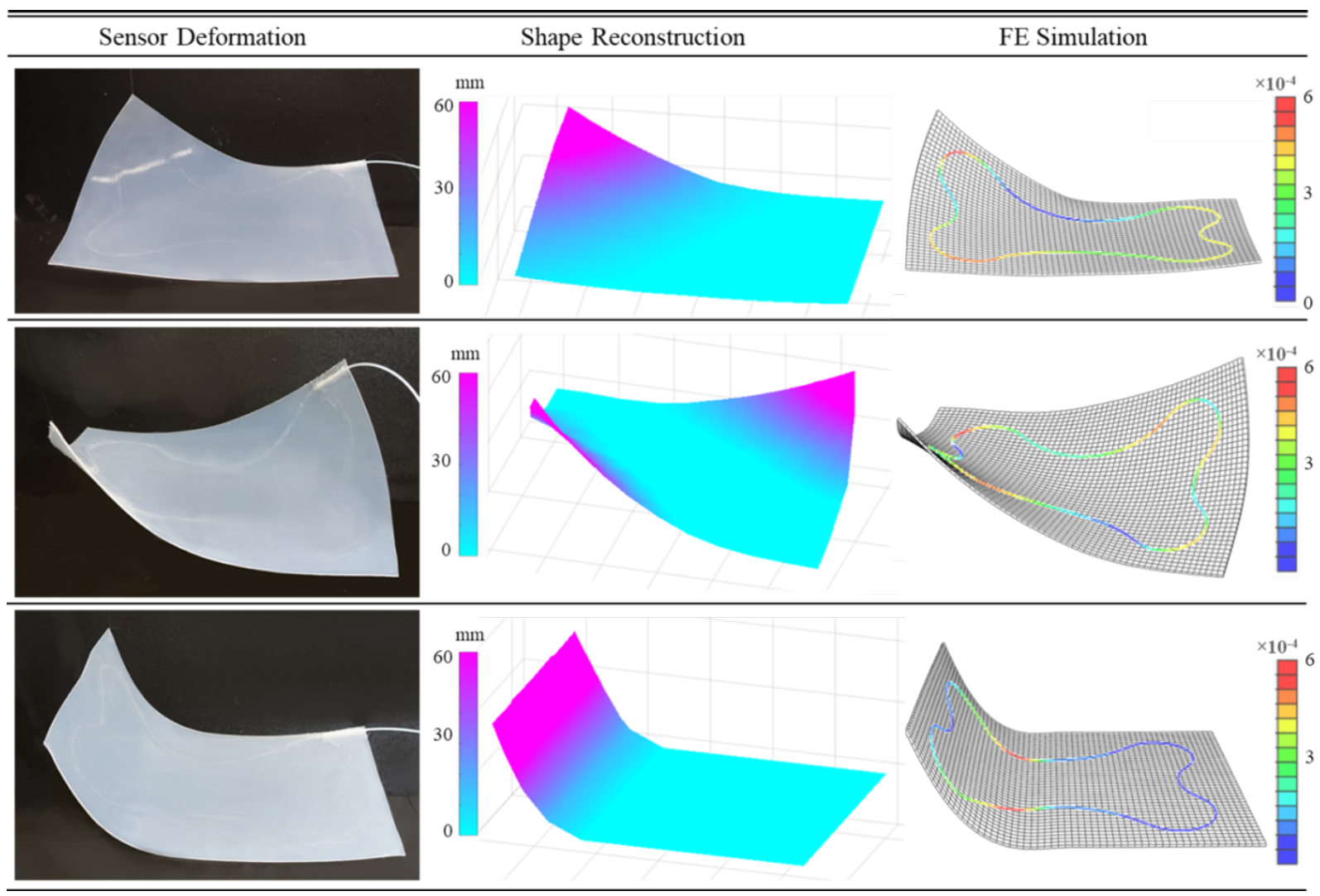

Figure 1: Fig. 1. Surface shape reconstruction instances. The deformed shape sensor (left column) is illustrated with corresponding shape reconstruction (middle column) with warmer colors indicating larger displacement (See Video 1, 2). FE simulations with fiber placement (right column) are shown with colored lines indicating the magnitude of fiber strain.

\section{Morphology Reconstruction via Ensemble Learning}

Proprioception in real-time typically requires high update frequency and low latency while minimizing compromise on sensing accuracy. To achieve high-frequency calculation, an ensembled model was used to create a mapping from the $29 \mathrm{FBG}$ strain data to a grid of $11 \times 7$ surface node positions evenly distributed along the shape sensor (Figure. 2a). 
a

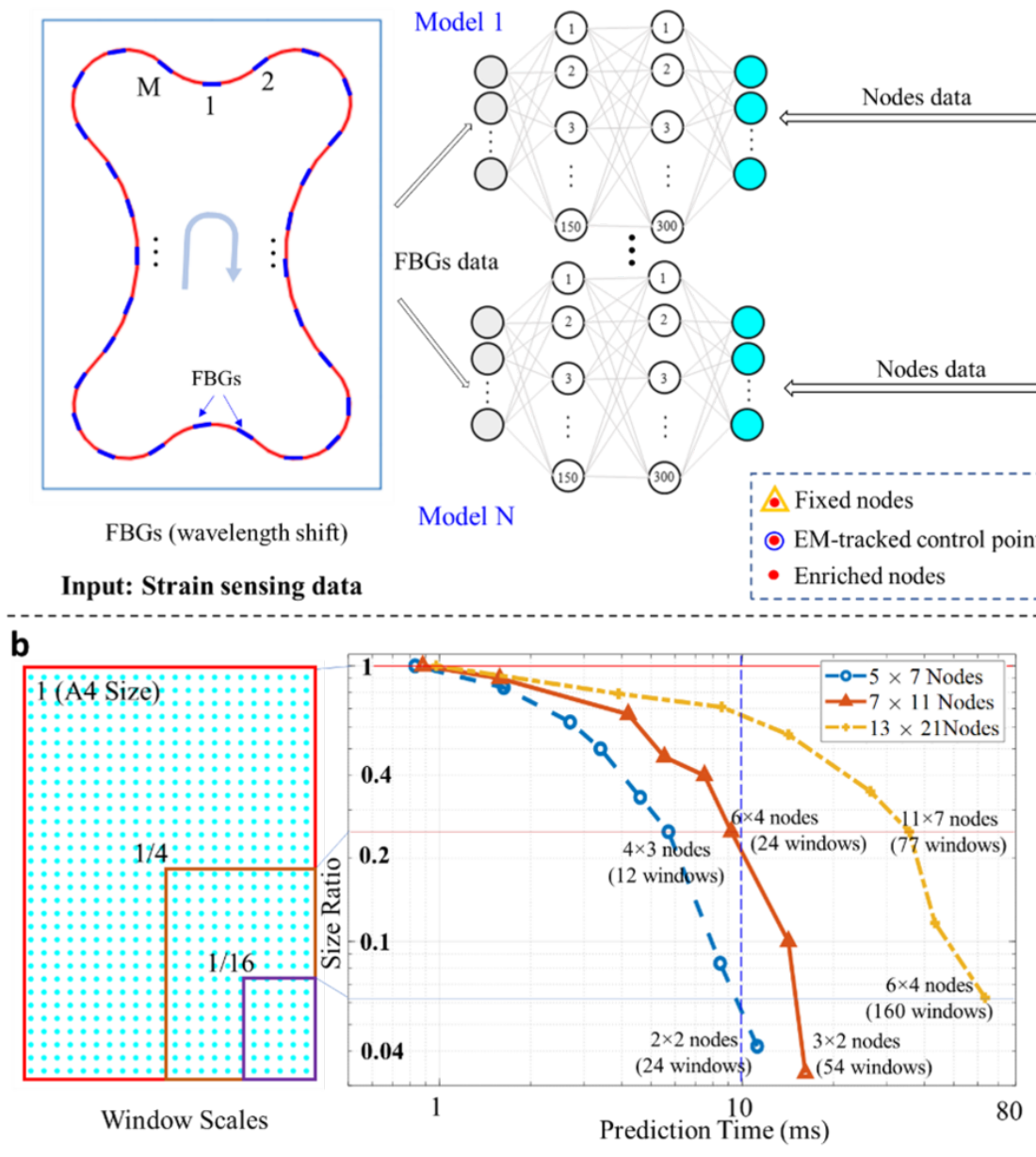

Window 1 Window 2

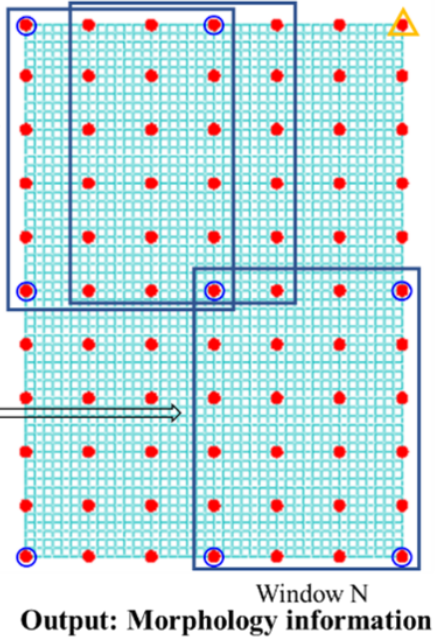
-1 Output: Morphology information

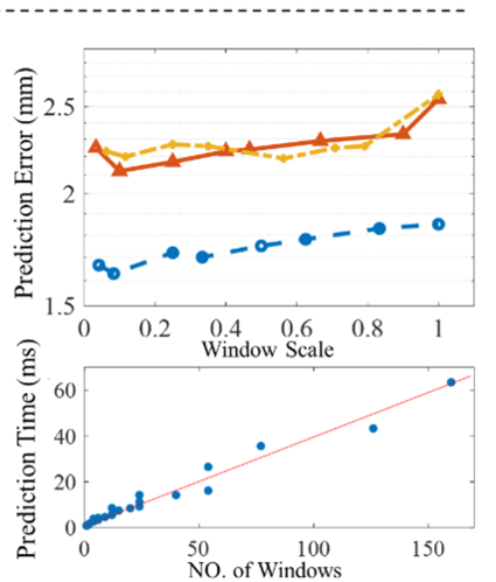

Figure 2: Fig. 2. Model training with ensemble learning. a, Model training with M Bragg shifted wavelengths as input to $\mathrm{N}(6 \times 4)$ multi-layer Perceptron regressors, which includes two hidden layers of 150 and 300 neurons, respectively. The whole surface nodes set is divided by $\mathrm{N}$ windows, with each window covering the same number of nodes. Each window involves an ANN-based sub-model with the same M (29) input FBGs data. b, Prediction time and accuracy as a result of changing the node density $(7 \times 5,11 \times 7$ and $21 \times 13$ nodes $)$ and the window size (from 1/16 to 1 times the A4 size), ), where the size ratio refers to the ratio of window size to the A4 size.

Ground truth data was obtained by capturing the 3-D position of nine control points selected on the shape sensor surface, which were distributed evenly along its four edges, along with one located in the center. During the data capture, one corner control point was fixed in position and the remaining eight control points were each measured at $40 \mathrm{~Hz}$ with 5-DoF electromagnetic (EM) tracking markers (Aurora $\mathbb{R}$ V3, NDI) attached to the shape sensor surface (Figure. 2a), while the FBG strain data was simultaneously captured (FBG-Scan 804D). All the data for model training was obtained in a single session of hands-on sensor deformation, which took over 100 seconds and 20 different 'key-frame' poses. The nine tracked control points were enriched to a grid of $11 \times 7$ positional nodes through FE-based data enrichment, and then used for the ensemble model output. The 29 strain-measuring FBGs were integrated into the shape sensor in a single optical fiber placed in a dog-bone layout (Figure. 2a), with center-to-center spacings of approximately $22 \mathrm{~mm}$ between FBGs. A total of 1,500 sets of FBG and node data were captured, with 1,000 used for training the learning model, 
200 for model validation, and 300 for testing of reconstruction accuracy. To withstand repeated strains sensing, we intentionally kept a safety threshold of bending radius during data acquisition. Extreme robot configurations that induce large local strains were avoided, and the resulting valid minimum sensing bending radius is approximately $20 \mathrm{~mm}$.

Ensemble learning configuration parameters in terms of (i) the node density (total nodes in the A4 size) and (ii) the sub-model size (window size) were tested to evaluate their effect on prediction accuracy and processing time. As shown in Figure. 2b, three different node densities (i.e., $7 \times 5,11 \times 7$ and $21 \times 13$ nodes) and eight window sizes (ranging from 1/16 to 1 times the A4 size) were compared. As for a specified node density, smaller window sizes would result in more windows (sub-models) for prediction, thereby increasing the processing time. It also reduces the prediction error, which refers to hereinafter as the deviation between the predicted nodal displacement and the EM-tracked displacement. The denser the node on the A4, the longer the prediction time due to the increased number of sub-models as well as the higher output data size for each sub-model. To maintain a reasonably high update frequency at $>100 \mathrm{~Hz}$ required for closed-loop robotic control, we have to limit the processing time per time step $<10 \mathrm{~ms}$; therefore, $11 \times 7$ nodes with 24 sub-models $(6 \times 4$ nodes) was selected for this A4-size surface model training (i7-6820HK, 16GB RAM, GTX 1070), taking account of a trade-off among the FE-based nodes density, the learning-based prediction error and the sensing frequency.

\section{- FE-Based Data Enrichment}

The integration of FE-based data enrichment highly relaxes the amount of ground truth that needs to be captured in a data-driven approach, where position-tracked control points data were imported into the FE model as displacement constraints to generate a rich amount of surface nodes data offline. Although we utilized an EM-based tracking system for ground truth capturing, other tracking modalities can be used with this sensing framework, provided that they are capable of accurately measuring node data on the shape sensor surface even in the case of complex or overlapping deformation. For example, camera-based motion capture systems can be used to obtain the original node data and then become enriched via FE modeling, thus reducing the number of motion reflective markers needed.(Rendl et al., 2014; Saunders et al., 2011; Dobrzynski et al., 2011)

Simulated environment was used to evaluate the accuracy of the FE-enrichment method in comparison to two commonly used surface approximation methods (Figure. 3a), namely bilinear and non-linear interpolations. A virtual A4-sized sensor was placed on a reference cylinder object (with radius of $\mathrm{R}=115 \mathrm{~mm}$ ), which acted as the ground truth shape. Each method used the 9 tracked sensor nodes as inputs/query points and displacement errors were calculated against the cylinder ground truth. The FE-enrichment method demonstrated a maximum displacement error of $3.2 \mathrm{~mm}$, which outperformed the piecewise bilinear interpolation method (max. error $\sim 19.7 \mathrm{~mm}$ ) and triangle-based nonlinear surface interpolation (max. error $~ 16.8 \mathrm{~mm}$ ). (Cuomo et al., 2014)The performance of the FE-based data enrichment method can be attributed to its use of the real sensor's geometric and material properties in the FE model. 

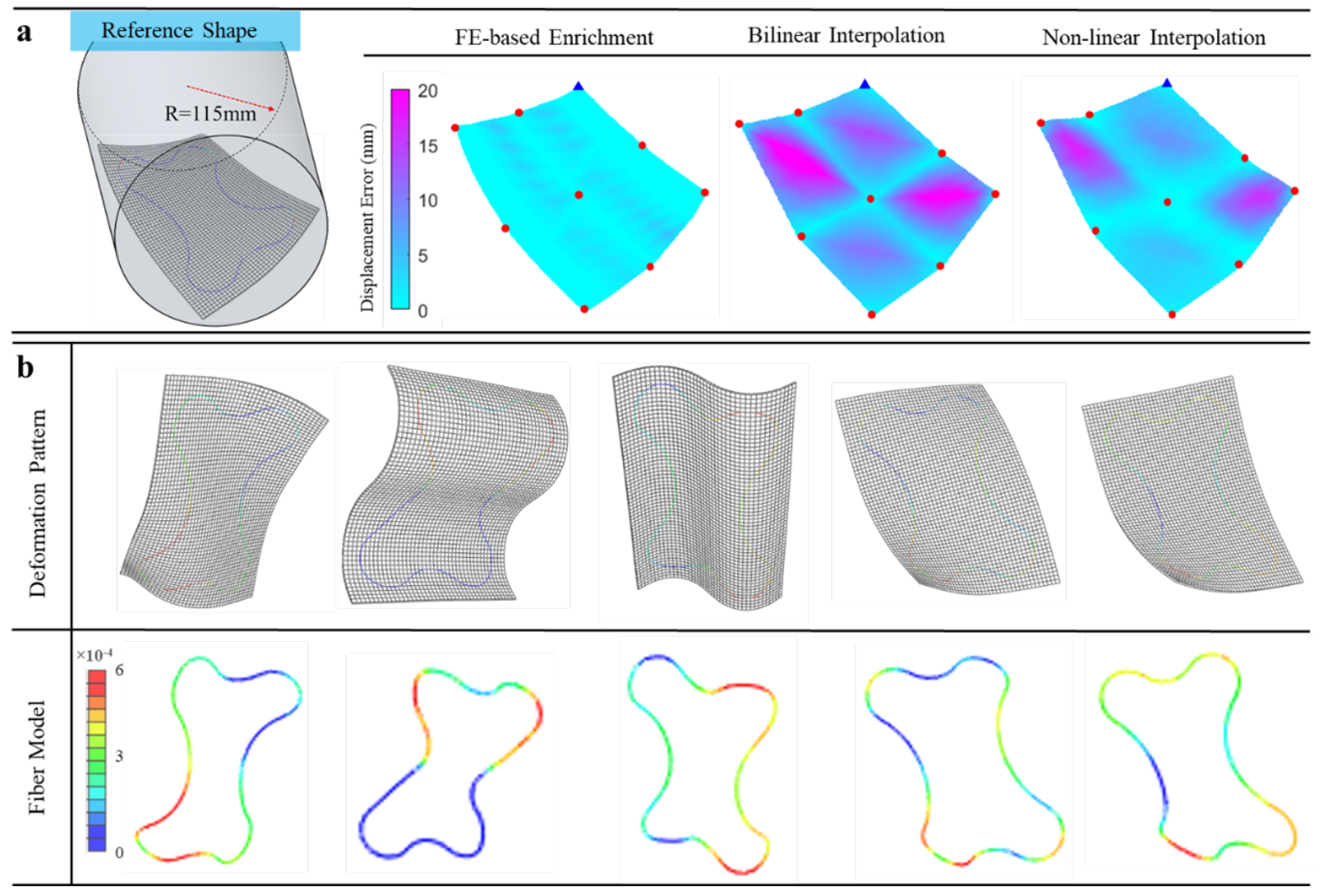

Figure 3: Fig. 3. FE model and surface approximation. a, Surface reconstruction with three approximation methods based on nine tracked nodes, indicated as one blue triangle (fixed nodes) and eight red circles (free nodes). The displacement errors of piecewise bilinear interpolation, triangle-based non-linear interpolation and FE-based data enrichment are color-coded with blue indicating a smaller error. b, Fiber strain responses of five deformation patterns in FE simulation, where warmer colors indicate a higher induced strain.

FE simulation for model training. The overall error was small with a mean of $0.6995 \mathrm{~mm}$, achieving a high goodness-of-fit with a correlation coefficient $>0.999$. The results support the feasibility of using a data-driven method to model the strain-morphology mapping prior to its real sensor fabrication, and the clear variation in fiber strains indicates that surface morphology could be well-differentiated and thus reconstructed from the strain data.

\section{- Accuracy and Flexibility}

The shape sensor flexibility was tested with various high-order deformation such that the surface nodes would undergo large displacements (Figure. 1 and Video 1). To evaluate shape sensing accuracy, 300 deformation instances were randomly selected to compare with the EM-tracked ground truth nodes. Note that such distinct deformation instances were not used in the previous model training. A histogram of 2,700 nodal displacement errors $(300 \times 9$ nodes $)$ is shown in Figure. 4a, which indicates that nearly $90 \%$ of the samples were well below $5 \mathrm{~mm}$ with a mean of $2.28 \mathrm{~mm}$. The root-mean-square error (RMSe) of each tracked node and the fixed point (A7), with respect to their position on the sensor, is illustrated in Figure. $\mathbf{4 b}$ and Figure. 4c. The warmer color of dots indicates a larger displacement sensing error. It can be observed that the prediction error of each node has a strong relationship with its distance to the fixed point and the mean displacement it underwent (Figure. 4c). The nodes A1, K1 and K7 have the largest error, likely because they have a higher degree of freedom, and hence underwent larger displacement. 


\section{- Repeatability and Hysteresis}

In this test, rather than only fixing a corner, a shorter edge of the shape sensor was clamped (Figure. 4d), enabling a larger degree of deformation along the long edge. The sensor was bent upward, downward, then back to the neutral position, counting as one bending cycle. The bending motion was generated by a linear actuator driving the distal edge (nodes K1 to K7) vertically, in order to carry out the sensing hysteresis test (Figure. S2). 

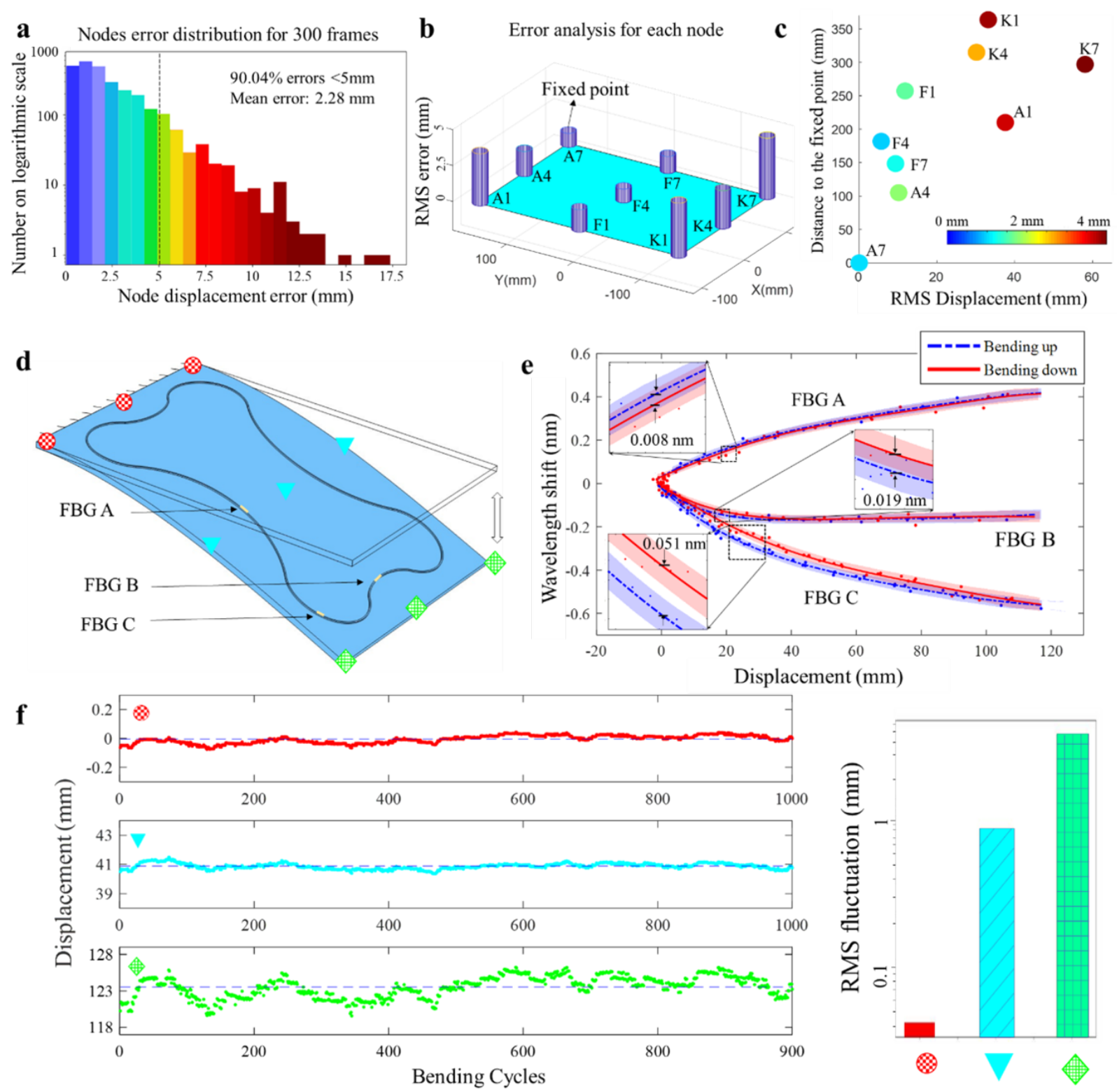

Figure 4: Fig. 4. Accuracy, repeatability and hysteresis analysis. a, Displacement error through 2,700 node instances samples collected from 300 frames. b, The corresponding room-mean-square error of each tracked node displacement. c, Error distribution compared with the distance from the fixed point (A7) and the node displacement. d, Setup for hysteresis and repeatability tests. The short edge of the shape sensor is clamped, with a linear actuator lifting the distal edge vertically. e, Hysteresis plot of three FBGs $A, B$ and $C$ under bending cycles $(0.5 \mathrm{~Hz})$, by fitting curves through sampling points. f, Peak displacement of the three sides, i.e., the clamped side (red), the middle line (cyan), and the distal side (green). The sensor showed high repeatability during 1,000 bending cycles. Quantitative comparison of the displacement fluctuation on a logarithmic scale (right).

As in Figure. 4e, three representative FBGs were selected, of which the Bragg wavelength shifts were read against the variation of distal edge displacement. Each data point was sampled by taking an average of three 
bending cycles. By fitting a curve along with those sampling points, the hysteresis could be measured as the disparity between the upward and downward bending, with the shadowed region representing the $95 \%$ confidence interval. Each hysteretic (or bending) cycle took around $2 \mathrm{~s}$. These disparities could be varied, depending on the FBG locations. In general, they are very small $(<0.051 \mathrm{~nm})$, thus implying a low level of sensing hysteresis throughout such large bending cycles. To evaluate the longer-term sensing repeatability, the displacements of three selected lines at the peak of upward motion were calculated and shown in Figure. 4f. Altogether, the results suggest that the sensing was still promising with stable and reliable responses over 1,000 repeated bending cycles. Again, this repeatability could also be varied by the displacement locations. The larger bending displacement detected by our shape sensor, the higher its fluctuation found over such repeated cycles. The fluctuation among those three lines of displacement nodes can be readily comparable on a logarithmic scale. Note that the nodes on the distal side encountered the largest fluctuation (RMS fluctuation $\sim 1.48 \mathrm{~mm}$ ) as a result of its largest displacement detected.

\section{- Underwater Test}

To further demonstrate the potential of our data-driven approach, we tested our training model in an underwater environment. A manta ray-shaped prototype was fabricated using the same settings of FBG fiber, following a similar procedure with the previous A4-sized design (Figure. 4a). An optical fiber with 29 FBGs was adhered roughly along the edges of the prototype (Figure. 5a), and its FE model is shown in Figure. 5b, where the red nodes represent the location of 8 EM tracking coils for the model training (ground truth). The optical fiber layout for the manta ray prototype is not a dog-bone shape, but a specific one based on its geometry. When we were dealing with this case with irregular shape, we did not just simply copy the fiber layout from the rectangular one (A4-size). The middle fixture of the ray prototype has divided the substrate into two parts such that the resulting possible sensor configuration is fewer than that of the A4 size prototype. When displaced by hydrodynamic force, the deformation magnitude and degree of freedom of the fin edge are larger than that of the inner region. Therefore, we adhered the optical fiber along the edge of the manta ray prototype. Upon vertical actuation $(1 \mathrm{~Hz})$, the manta ray was displaced vertically underwater. The sensing performance of the manta ray fins can be referred to in Video $\mathbf{3}$ as well as Figure. 5, where Figure. 5c shows the ray at three typical deformation instances and Figure. 5d illustrates their corresponding real-time shape reconstruction. The ever-changing water drag did not hinder the sensing performance even with the model training and data-driven analysis initially conducted in the air. 


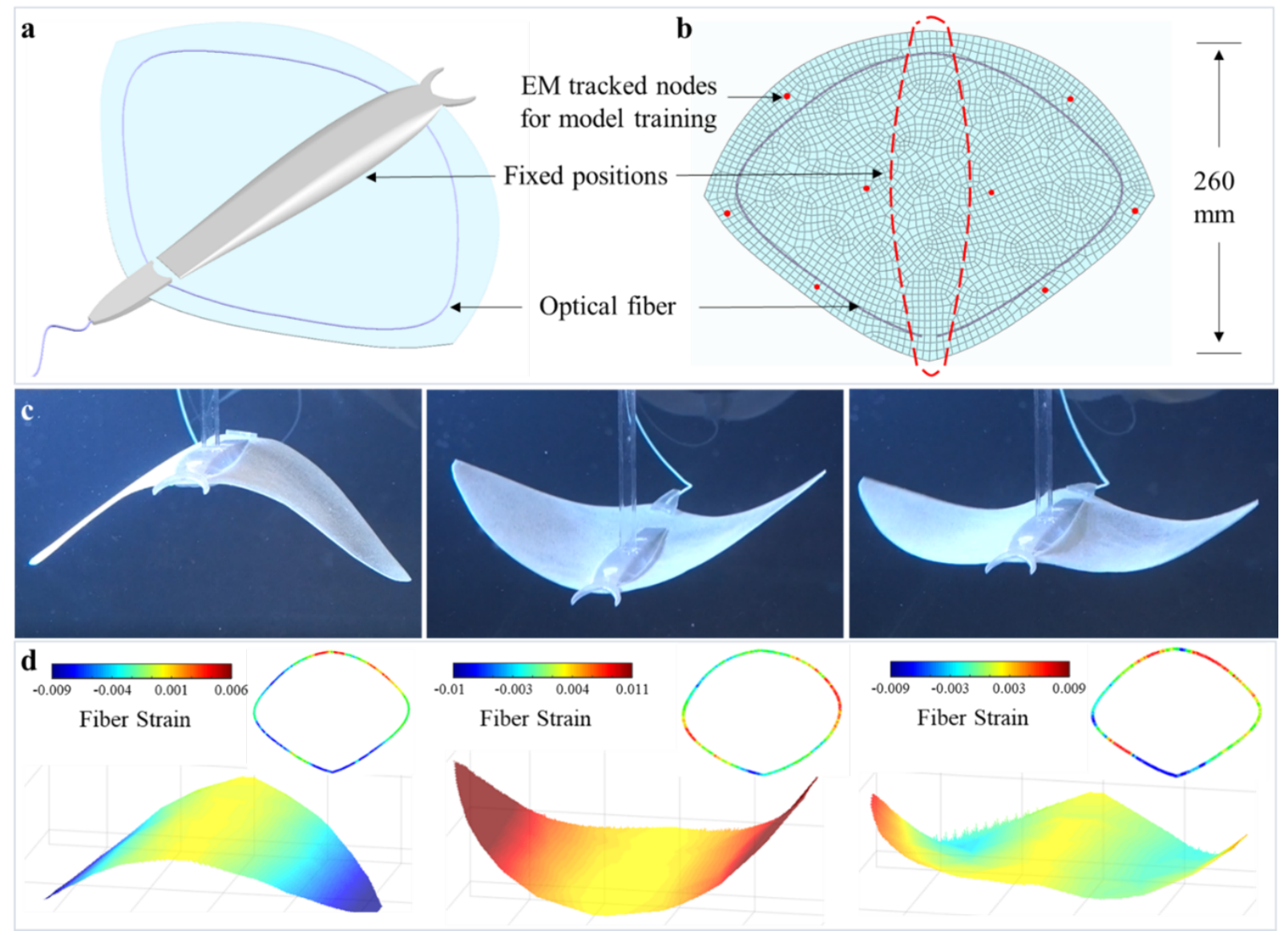

Figure 5: Fig. 5. Shape sensing of underwater manta ray fin. a, Overview of the fin profile, with its FE model shown in b. The soft fin made of silicone rubber was moved by a vertically actuated rod, interacting with continuous hydrodynamic forces. c, Three different phases of deformation poses and their corresponding shape reconstructed in real-time as in d and Video 3, Warmer the color, higher the fiber strain induced along the fin contour. Note that the learning model training was conducted out of water beforehand. The unnoticeable deformation asymmetry can be reflected by its strains measured. 


\section{Supplementary Figure S1}

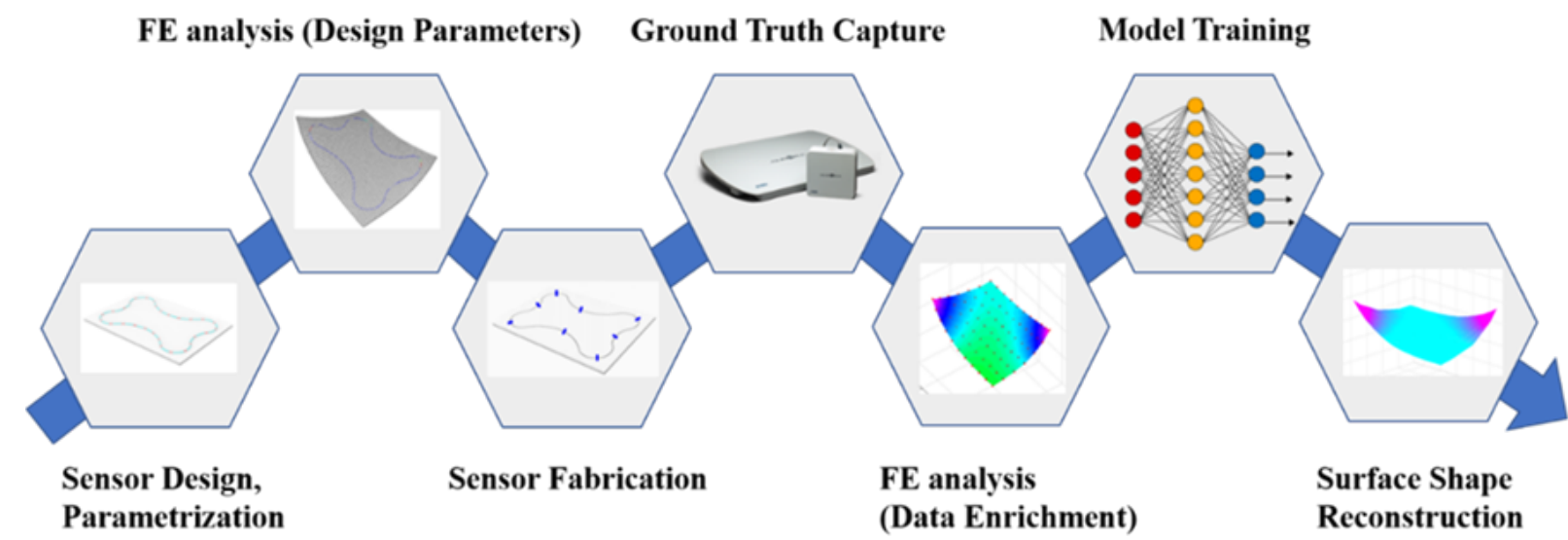

Figure 6: Figure S1. Workflow of the proposed surface shape sensing framework. FE analysis plays a fundamental part in the proposed framework and is utilized in the sensor design stage to optimize design parameters prior to fabrication. After fabrication and ground truth capture, FE analysis is used to enrich the input data for model training and allow high-density and real-time surface shape reconstruction. 


\section{Supplementary Figure S2}
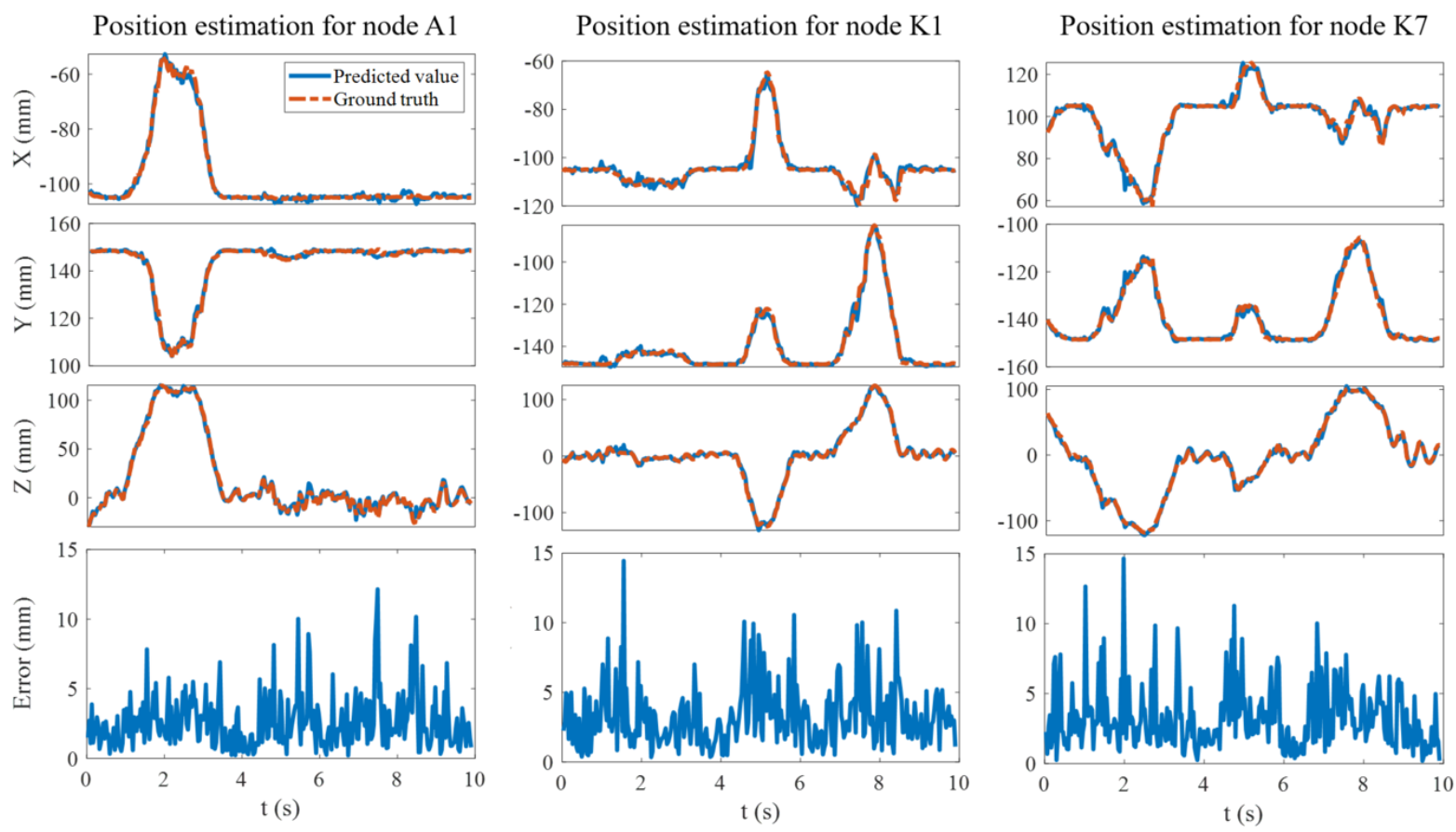

Figure 7: Figure S2. Positions estimation with the reconstruction algorithm. The predicted position (solid blue) for three nodes (A1, K1, K7) was compared with their corresponding ground truth (dashed red) during a period of 10 s continuous deformation. The last row shows the prediction error during the deformation, which indicates that most of the predicted error was less than $5 \mathrm{~mm}$. 


\section{Supplementary Figure S3}

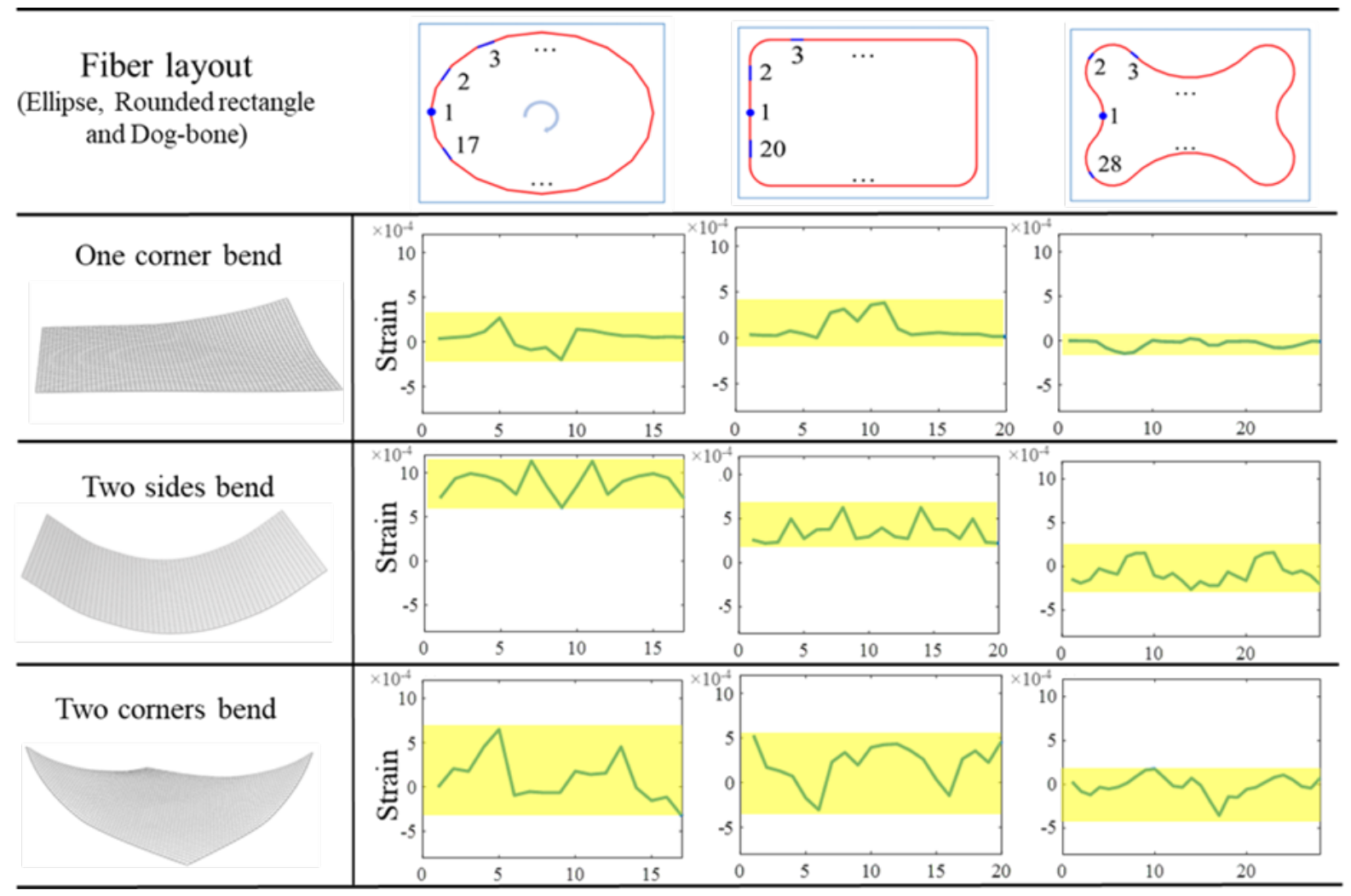

Figure 8: Figure S3. Strain response comparison between three fiber layouts. Assuming that FBGs are distributed in the clockwise direction, the strain responses of three typical fiber layouts are analyzed with FEA, including elliptical, rounded rectangle and dog-bone layouts. Three typical deformation patterns are simulated to compare the strain response. Peak-to-peak strain is highlighted in yellow. 


\section{Supplementary Figure S4}

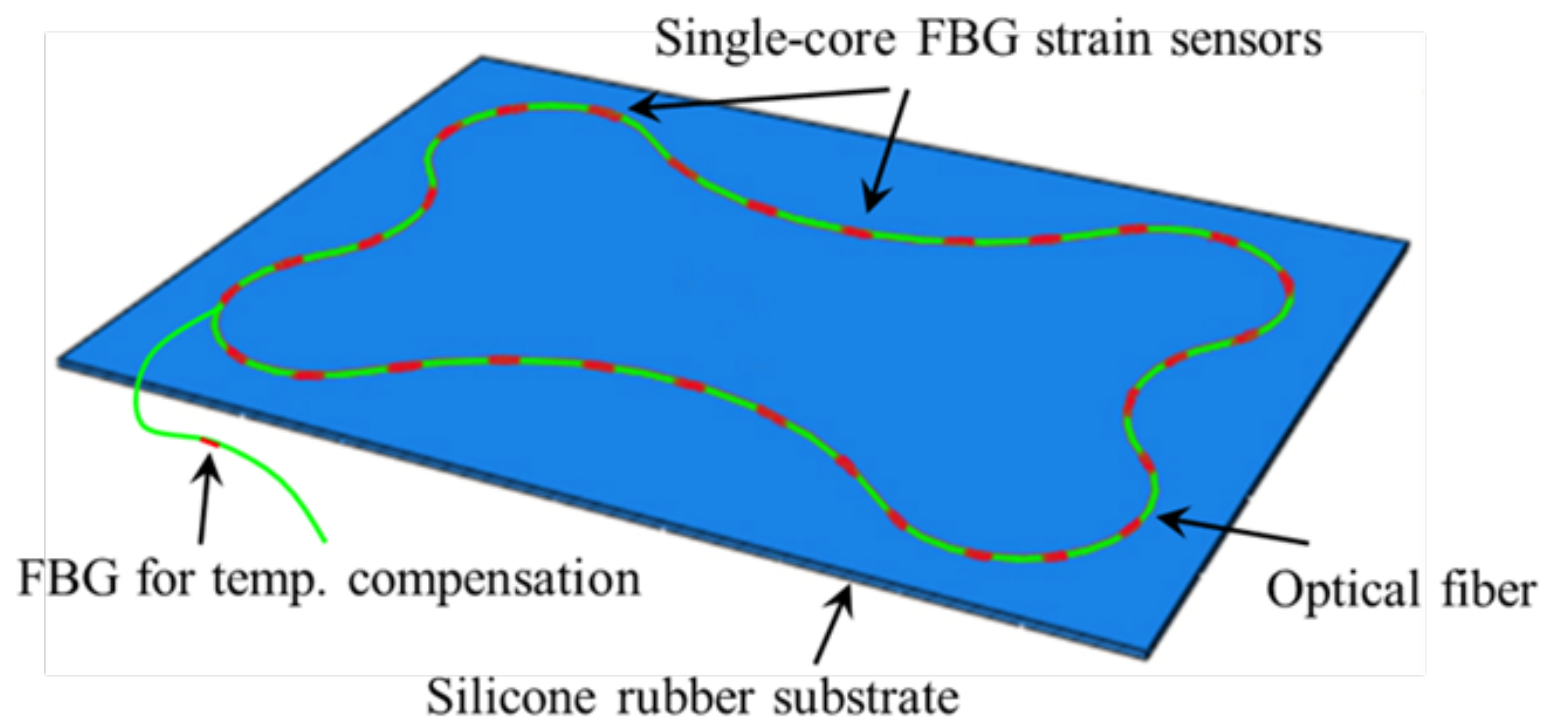

Figure 9: Figure S4. Flexible shape sensor prototype in A4 size. An optical fiber with 28 sparse FBG strain sensors was attached on the surface of the silicone rubber substrate, following a dog-bone path (See Video 5). FBGs are sparsely distributed along the fiber, measuring the local strains for global morphological estimation. 


\section{Supplementary Table S5}

\begin{tabular}{|c|c|c|c|c|c|}
\hline Research & This work & $\begin{array}{l}\text { Y. L. Park, } \\
\text { et al. [52] }\end{array}$ & $\begin{array}{l}\text { T. L. T. Lun, } \\
\text { et al. [19] }\end{array}$ & $\begin{array}{l}\text { G. Cheng, } \\
\text { et al. [11] }\end{array}$ & $\begin{array}{l}\text { C. Rendl, } \\
\text { et al. [17] }\end{array}$ \\
\hline Dimensions & $\begin{array}{c}297 \times 210 \times 1 \\
\mathrm{~mm}\end{array}$ & $\begin{array}{c}25 \times 25 \times 3.5 \\
\mathrm{~mm}\end{array}$ & $\begin{array}{c}84 \times 84 \times 5 \\
\mathrm{~mm}\end{array}$ & $\begin{array}{c}28 \times 28 \times 3.5 \\
\mathrm{~mm}\end{array}$ & $\begin{array}{c}210 \times 130 \times 1.175 \\
\mathrm{~mm}\end{array}$ \\
\hline $\begin{array}{c}\text { Sensing } \\
\text { unit }\end{array}$ & FBG & $\begin{array}{l}\text { Liquid metal } \\
\text { (EGaIn) }\end{array}$ & FBG & $\begin{array}{c}\text { Triaxial } \\
\text { accelerometer }\end{array}$ & $\begin{array}{l}\text { Piezoelectric bend } \\
\text { sensor }\end{array}$ \\
\hline $\begin{array}{l}\text { Modelling } \\
\text { method }\end{array}$ & $\begin{array}{c}\text { Ensemble } \\
\text { Learning }\end{array}$ & $\begin{array}{l}\text { Theoretical } \\
\text { model }\end{array}$ & $\begin{array}{l}\text { Artificial neural } \\
\text { network }\end{array}$ & $\begin{array}{l}\text { Relative } \\
\text { rotation } \\
\text { estimation }\end{array}$ & $\begin{array}{l}\text { Learning-based } \\
\text { Continuous } \\
\text { Regression }\end{array}$ \\
\hline $\begin{array}{l}\text { Sampling } \\
\text { rate }\end{array}$ & $>100 \mathrm{~Hz}$ & $50 \mathrm{~Hz}$ & $30 \mathrm{~Hz}$ & $4 \mathrm{~Hz}$ & NA \\
\hline Accuracy & $\begin{array}{l}\text { RMS error of } \\
\text { nodal positions } \\
\sim 2.28 \mathrm{~mm}\end{array}$ & $\begin{array}{l}\text { "RMS } \\
\text { displacement } \\
\text { errors of } 1.6 \mathrm{~mm} \\
\text { in the x-axis and } \\
1.8 \mathrm{~mm} \text { in the y- } \\
\text { axis." }\end{array}$ & $\begin{array}{l}\text { "The sensor } \\
\text { achieved an } \\
\text { accuracy of RMSe } \\
=1.17 \mathrm{~mm} \text { for } \\
\text { reconstructed node } \\
\text { displacements" }\end{array}$ & $\begin{array}{l}\text { "Loop length } \\
\mathrm{L}=222.28 \\
\mathrm{~mm} \text {, the error } \\
\text { is } 4.71 \% "\end{array}$ & $\begin{array}{l}\text { "The learning } \\
\text { methods achieve } \\
\text { the best results } \\
\text { with an average } \\
\text { error of } 0.015 \pm \\
0.007 \mathrm{~m} "\end{array}$ \\
\hline
\end{tabular}

Figure 10: Table S5. Comparison of several representative flexible skins.

\section{Supplementary Video S1}

Rich media available at https://youtu.be/togFKD9SAtA

\section{Supplementary Video S2}

Rich media available at https://youtu.be/uvHbG1b92pU

\section{Supplementary Video S3}

Rich media available at https://youtu.be/ROJ_gSbsTiM

\section{Supplementary Video S4}

Rich media available at https://youtu.be/rEUApmnSzIs

\section{Supplementary Video S5}

Rich media available at https://youtu.be/Jw-IU_Fb5uA 


\section{References}

FlexSense. (2014, October). Proceedings of the 27th Annual ACM Symposium on User Interface Software and Technology. https://doi.org/10.1145/2642918.2647405

Experimental verification of soft-robot gaits evolved using a lumped dynamic model. (2011). Robotica, 29(6), 823-830. https://doi.org/10.1017/s0263574711000014

Contactless deflection sensor for soft robots. (2011, September). 2011 IEEE/RSJ International Conference on Intelligent Robots and Systems. https://doi.org/10.1109/iros.2011.6094845

A novel triangle-based method for scattered data interpolation. (2014). Applied Mathematical Sciences, 8, 6717-6724. https://doi.org/10.12988/ams.2014.49686 